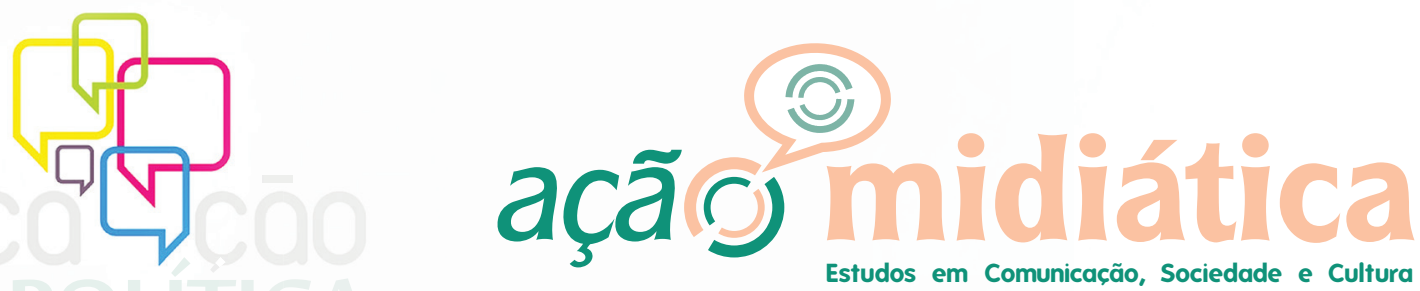

Estudos em Comunicação, Sociedade e Cultura

N. 6 | Ano 2013

\title{
Redes de acción pública en México. Una breve historia ${ }^{1}$
}

As redes de ação pública no México. Uma breve história

Networks of public action in Mexico. A brief history

Edgar Esquivel SOLÍS ${ }^{2}$

\begin{abstract}
RESUMEN
El presente trabajo hace una breve historización del surgimiento de redes de organizaciones civiles que promovieron con su trabajo la atención de diversos temas como la agenda de derechos humanos, la de género y la atención a temas vitales como la vivienda, la dotación de servicios públicos. El reconocimiento de que el trabajo en red les permitía a estas organizaciones cobrar fuerza frente al Estado mexicano les permitió desarrollar estrategias de defensa en temas comunes y ante los excesos y abusos de éste y así mismo el compartir expertise, conocimientos, estrategias comunes e información.
\end{abstract}

Palabras clave: Nuevos actores sociales; Sociedad civil; Redes de organizaciones civiles; México.

\section{RESUMO}

O presente trabalho realiza uma breve contextualização histórica do surgimento de redes de organizações civis que promoveram debate sobre diversos temas como direitos humanos, gênero e temas essenciais como moradia, distribuição de recursos públicos. O reconhecimento de que o trabalho em rede permitia a essas organizações reivindicar apoio ao Estado mexicano proporcionou desenvolver estratégias de defesa de causas comuns e, diante dos excessos deste, assim mesmo, compartilhar know how, conhecimentos, estratégias comuns e informação.

Palavras-chave: Novos atores sociais; Sociedade civil; Redes de organização civil; México.

\begin{abstract}
This paper provides a brief historical overview of the emergence of networks of civil organizations that promoted discussion on various topics such as human rights, gender and key issues such as housing, distribution of public resources. The recognition that networking allowed these organizations claim to support the Mexican State, provided develop strategies for defending common causes and, before the excesses of this, anyway, share expertise, knowledge, common strategies and information.
\end{abstract}

Keywords: New social actors; Civil society; Networks of civil organization; Mexico.

\section{Introducción}

El tema de las redes de acción pública como tema de análisis ha cobrado un particular interés en la última década. El desarrollo de ellas ha ido de la mano del impulso mismo y creación de organizaciones sociales. Al igual que se registró en los años noventa la generación

1 Trabalho apresentado à sexta edição da Revista Ação Midiática - Estudos em Comunicação, Sociedade e Cultura, publicação ligada ao Programa de Pós-Graduação em Comunicação, da Universidade Federal do Paraná.

2 Profesor-investigador de la Universidad Autónoma Metropolitana-Cuajimalpa, México. 


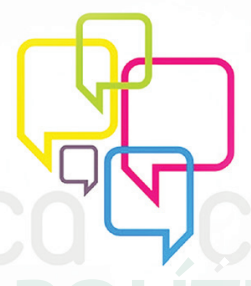

açãô I0 mididiática

Universidade Federal do Paraná | Programa de Pós-Graduação em Comunicação

del mayor número de organizaciones de la sociedad civil se desarrollaron redes de organizaciones temáticas.

Las redes se han desarrollado para propósitos muy diversos, consecuentemente sus temas reflejan las preocupaciones - y temas - en torno de los actores que se conjuntan. Existen redes de políticas públicas (LUNA, 2005; CAMPOS, 2003), hay también redes en torno de la lucha por la democracia (OLVERA, 2004; CADENA, 2004). También se han desarrollado redes denominadas epistémicas, al vincular a diversos actores en torno de especializaciones diversas.

El objetivo de estas líneas es destacar la importancia de dichas redes por sus contribuciones a la sociedad mexicana que han girado en torno de:

a) impactan y promueven el desarrollo en comunidades rurales de alta marginación y pobreza. El papel de la participación ciudadana como el componente primordial de estas redes es de vital importancia ya que logra colocar en las agendas de gobierno sus necesidades primarias (servicios públicos: agua, drenaje, alcantarillado, electrificación) mismas que, al ser gestionadas a través de redes de políticas públicas (RPP) consecuentemente fortalecen la legitimidad de la política, al ser procesada horizontalmente.

b) Pluralizar - democratizando - a la sociedad mexicana, fortaleciendo el reconocimiento del carácter diverso en la conformación de la misma y fomentando la tolerancia en una sociedad que se ha ido diferenciando gradualmente;

c) Promover temas a través del intercambio horizontal de información y recursos además de la interacción de diversos actores que rebasan el marco de la sociedad civil para fomentar la cooperación intersectorial.

d) Generar un capital humano (expertisse) en los liderazgos de las organizaciones que permite que a través de diversos recursos (generación de opinión pública y cabildeo) se propongan leyes y políticas públicas específicas.

e) Transformar algunas prácticas lesivas - p.e. marginación, exclusión e intolerancia - de la sociedad al igual que del sistema económico y político, esto, a través de multiplicar los canales de acceso a grupos que carecen de representación o que atienden temas muy específicos (niñez, minusválidos, vejez, madres solteras, etc).

Desarrollaremos primeramente una breve discusión sobre las redes conceptualmente, es decir como un fenómeno social. Posteriormente presentamos una historización crítica del desarrollo de las redes de organizaciones civiles en México y su contribución al cambio social en México. 


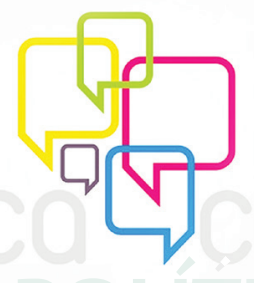

açãô Imidiática

N. 6 | Ano 2013

\section{Universidade Federal do Paraná I Programa de Pós-Graduação em Comunicação}

\section{Las redes como concepto}

Los estudios iniciales de redes sociales se ubican en la antropología inglesa en la década de los cincuenta en el pasado siglo XX ( $c f r$. Radcliffe-Brown). En México dentro de los estudios señeros bajo este enfoque teórico se localiza el trabajo de Larissa Lomnnitz (2006), quien en su estudio Cómo sobreviven los pobres, presentado en los años setenta, detalla como los grupos de actores más pobres del sistema desarrollan estrategias de sobrevivencia a través de interacciones horizontales, y afincadas principalmente en intercambios de bienes materiales (sobre todo alimento) de manera recíproca.

Desde el ángulo de la sociedad civil existen diversas líneas como la que ofrece Sydney Tarrow (1997), quien desde el ángulo de la interacción e intercambio político (a través de lo que denomina como ciclos de protesta), señala que las redes de actores sociales participan de lo que él denomina un

"ciclo de protesta", me refiero a una fase de intensificación de los conflictos y la confrontación en el sistema social, que incluye una rápida difusión de la acción colectiva de los sectores más movilizados a los menos movilizados; un ritmo de innovación acelerado en las formas de confrontación; marcos nuevos o transformados para la acción colectiva; una combinación de participación organizada y no organizada; y unas secuencias de interacción intensificada entre disidentes y autoridades que pueden terminar en la reforma, la represión y, a veces, en una revolución (TARROW, 1997, p. 263-64).

Para Tarrow, la acción de las redes queda enmarcada por la creación de nuevos marcos de acción. Todo ello realizado por redes de actores sociales. Para Keck y Sikkink existen campañas orquestadas con propósitos muy específicos, que desarrollan diversos tipos de acciones (KECK y SIKKINK, 2000). Sin embargo para los propósitos de lo que aquí discutimos coincidimos con Jorge Cadena, para quien la idea de red es una vinculación horizontal en torno a valores y temas de interés en común a partir de los cuales los actores de la SC orientan acciones y promueven dinámicas, acciones y/o campañas. Jorge Cadena Roa las denomina redes de confianza y señala

\footnotetext{
Al entrar en contacto entre sí en actividades reivindicativas fueron ampliando las redes de confianza alternativas que facilitaron la cooperación entre ellas a partir del reconocimiento de la independencia y la autonomía de las organizaciones participantes en relación con quien era identificado como su principal interlocutor y oponente, el Estado autoritario mexicano (2004, p.158).
}

\section{Antecedentes}

El desarrollo de estas redes se registra a inicios del XX, en el sureste mexicano con el incipiente movimiento feminista en el estado de Yucatán. Para Anna Macías previo al 


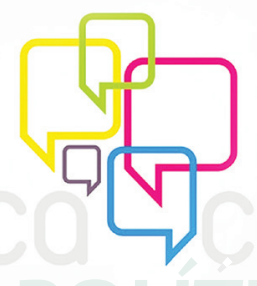

açãô Imidiática

N. 6 | Ano 2013

Universidade Federal do Paraná | Programa de Pós-Graduação em Comunicação

movimiento revolucionario se registraba ya un incipiente movimiento feminista encabezado principalmente por mujeres educadas, de clase media que exigían oportunidades de educación para todas, mejor paga y reformas al Código Civil (MACÍAS, 2002, p. 37).

En el desarrollo del feminismo fueron importantes las contribuciones que destacadas mujeres líderes e intelectuales realizaron. Muchas se concretaron por ejemplo en la propuesta de Venustiano Carranza de permitir el divorcio en nuestro país, iniciativa que fue apoyada por todos los estados entre 1915 y 1917, a partir de entonces se permitió volverse a casar. Posteriormente el presidente Lázaro Cárdenas introdujo la educación sexual en las escuelas públicas, demanda hecha durante años por feministas y a las que al igual que el divorcio se oponía firmemente la Iglesia católica (MACÍAS, 2002, p. 54-55). El feminismo de los años veinte y treinta desarrollaría así, un fuerte sentimiento anticlerical.

El sureño estado de Yucatán experimentó importantes avances para el movimiento feminista. Se realizaron dos congresos feministas en su capital, Mérida, mismos que serían los primeros realizados en nuestro país. El estado reunía condiciones singulares, una etapa de esplendor económico con el cultivo y demanda del henequén, un servicio de buques de vapor que llegaban a Estados Unidos (Nueva York y Nueva Orleáns) y la Habana, lo cual imprimía un aire cosmopolita a la capital.

El apoyo al movimiento feminista provino primeramente del gobernador Salvador Alvarado, quien patrocinó el primer congreso feminista realizado en nuestro país, segundo en América Latina (el primer país en organizarlo fue Argentina). Anna Macías señala que para la liberación femenina en México, Yucatán se volvió un estado defensor de los derechos de las mujeres con los gobiernos de Salvador Alvarado y Felipe Carrillo Puerto (2002, p. 110).

Yucatán fue también el primer estado en México en aprobar el voto de la mujer. Tuvo dos diputadas. Carrillo Puerto compartía con Alvarado la idea de que las mujeres debían organizarse y con el apoyo de su hermana Elvia organizó ligas feministas en el estado.

En 1923, las feministas mexicanas tenían muchos obstáculos que superar. En sus esfuerzos por terminar con su exclusión del mundo y su inferioridad legal social y política, y por conseguir mayor libertad personal, tuvieron que enfrentar enemigos conservadores muy poderosos. Al mismo tiempo, tuvieron que marcar su distancia de ciertos defensores, como Felipe Carrillo Puerto, cuyas ideas radicales afectaban su causa (MACÍAS, 2002, p. 127).

La misma Anna Macías considera que el feminismo moderno en el mundo es en gran medida un fenómeno urbano, cuyas dirigentes tienen una educación superior a la media de la población en general y en México eso no fue la excepción. 


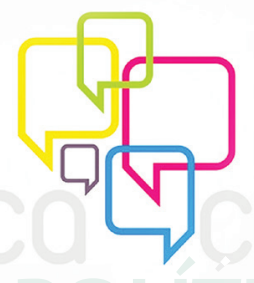

açãô Imidiática

N. 6 | Ano 2013

\section{Universidade Federal do Paraná I Programa de Pós-Graduação em Comunicação}

Se considera que con el reconocimiento del voto de la mujer en 1954 el movimiento entraría en una etapa de aletargamiento. Es sólo hasta los años setenta que encontramos, después de una especie de dilatamiento, el resurgimiento del MFM. Para Rosario Campos dos factores que posibilitaron este resurgimiento fueron: primero, la obtención del voto de la mujer, que para nuestro caso fue en 1954. Esta se considera la "primer ola del feminismo", que conecta plenamente con el movimiento sufragista transnacional. Posteriormente, la "segunda ola" se propició en torno a las relaciones entre hombre y mujeres con demandas específicas como: despenalización del aborto, el uso masivo de anticonceptivos (proporcionados desde el Estado), el ejercicio libre de la plena sexualidad, la demanda de la socialización de las tareas domésticas y la violencia sexual en contra de las mujeres. La "tercera ola", vino a significar el establecimiento de lineamientos para que los países adopten la perspectiva de género, a partir de conferencias y convenios internacionales (2003, p.26).

Frente al MFM el Estado mexicano ha tenido diferentes posturas

La Primer Conferencia Internacional de la mujer, realizada en nuestro país en 1975, y el Decenio de la Mujer que le sucedió fueron motivo de un acercamiento relativo hacia los grupos feministas para que participaran en la elaboración del Primer Informe sobre la Condición de la Mujer, presentado durante la conferencia (CAMPOS, 2003, p.35).

Para Alma Rosa Sánchez, la presencia organizada de las mujeres en los últimos quince años del siglo XX significó la reivindicación del derecho a la diferencia, a construir una identidad femenina como sujeto político que crítica el ejercicio del poder. Ella al MFM lo define como el movimiento amplio de mujeres (MAM) (SÁNCHEZ, 2004, p.88).

El MAM se construyó a partir de cinco sectores, que plantearon su participación en dos planos, su participación en la democracia y en la defensa de derechos para las mujeres. Para Sánchez Olvera estos cinco sectores serían:

a) El movimiento feminista; constituido principalmente por académicas, periodistas, escritoras, políticas, mujeres de clase media.

b) El movimiento urbano popular; con demandas materiales, servicios, vivienda, agua, drenaje, educación, entre otros, sector marginal urbano.

c) El movimiento de campesinas e indígenas; reclamos sobre acceso a crédito, propiedad, tecnología y capacitación.

d) El movimiento de trabajadoras asalariadas; ampliación de mercado de trabajo, mejoras salariales, denuncias de hostigamiento sexual, entre otras, y; 


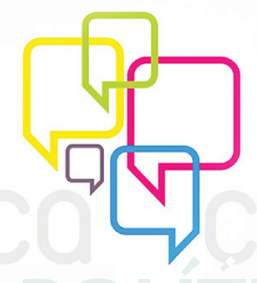

ação mididiática

N. 6 | Ano 2013

\section{Universidade Federal do Paraná I Programa de Pós-Graduação em Comunicação}

e) Mujeres militantes de partidos políticos y funcionarias; aquí se asumen como feministas y denuncian las condiciones desiguales de participación de la mujer en México (SÁNCHEZ, 2004, p.90-91).

En el sexenio de Carlos Salinas, fue creado el Programa Nacional de Solidaridad que en lo tocante al MFM la propia Rosario Campos considera que "se sentaron las bases entre el Estado y las organizaciones en el desarrollo de programas específicos ligados a las políticas públicas" (2003, p.37). En la administración de Ernesto Zedillo, aunque está fuera de nuestro periodo de análisis baste señalar que se creó la Comisión Nacional de la Mujer (CONMUJER), instancia que operaba bajo la dirección de la Secretaría de Gobernación.

La investigadora Adriana Sandoval sostiene al igual que Rosario Campos que hay elementos que a pesar del impulso del MFM desde este subperiodo (de hecho desde la aparición del mismo movimiento), dificultan aún e impiden la plena participación de la mujer en el espacio público. Específicamente y a contrapelo de las tesis de algunos indigenistas que idealizan la vida de dichas comunidades como sinónimo de "bondad y pureza" (frente al "perverso" mundo occidental) estas dos investigadoras (cada una por su cuenta) encuentran diversos elementos como

\footnotetext{
que la participación en las organizaciones comunitarias, es una participación costosa para las y los indígenas campesinos. Por el otro, la participación es diferencial, ya que no es homogénea, como tampoco los alcances de ésta, identificando diferencias de participación e inequidades en el acceso y control de los recursos, así como de los beneficios. Estas situaciones problemáticas en las interacciones generalmente se expresan como relaciones de inequidad (SANDOVAL, 2005, p. 346-7).
}

Existen críticas fuertes al MFM, que son de alguna forma las mismas que se le dirigen a la SC en su conjunto, y estas apuntan a lo limitado de su alcance frente a un proceso de pauperización, empobrecimiento y su resultante: la exclusión de poco más de la mitad de la población mexicana (si nos atenemos sólo a las cifras oficiales), agravada desde el viraje de modelo económico y en la conducción del Estado mexicano hace más de veinte años y desarrollado fuertemente desde este subperiodo (1984-1993).

María Luisa Tarrés apunta, al igual que otros investigadores que justo la carencia de un espacio institucional de encuentro entre autoridades y organizaciones de la sociedad civil (OSC) dificultó en este periodo el avance a partir del entendimiento conjunto entre las necesidades y demandas del MFM.

Para María Luisa Tarrés la relación entre la SC y el Estado, particularmente en nuestro país enfrenta uno de los más serios desafíos por la tensión entre una sociedad que 

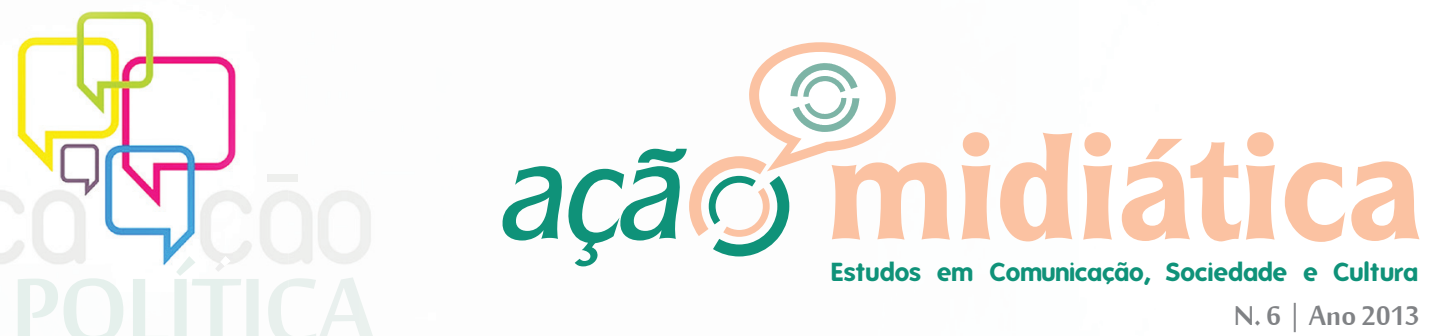

Universidade Federal do Paraná | Programa de Pós-Graduação em Comunicação

se ha modernizado y las tradicionales formas de organización política de las élites. Esto lo apunta al respecto de las organizaciones que se abocan a trabajar los temas de las mujeres en particular.

Esto mismo es señalado por diversos intelectuales (ARDITÍ, 2003; CROUCH, 2004 y PIZZORNO, 2005), quienes advierten del riesgo que entraña que amplios sectores de la población no se sientan representados ni atendidos por los políticos, encontrando en el mejor de los casos la atención de sus demandas y/o intereses específicos en la SC, concretamente en el mundo de las OSC y MS, incluso en un ángulo menos o nada conveniente en grupos radicales (del tipo skin heads, o pandillas trasnacionales: Mara Salvatrucha, entre otros), agregaríamos nosotros.

El feminismo como uno de los primeros movimientos que en la sociedad impulsó la creación de redes, al involucrar a una diversidad de actores en torno de las temáticas que ha promovido a lo largo de su existencia.

\section{Redes de promoción y defensa de los derechos humanos en México}

En la dinámica de los cambios sociales es de destacar la importancia de fenómenos acontecidos en el contexto internacional, como serían el incremento en el número e importancia organizaciones internacionales. Así, "la primera organización de derechos humanos que obtuvo amplio reconocimiento internacional fue Amnistía Internacional, formada en los sesenta. AI tomó algunas decisiones tácticas clave que sirvieron para enmarcar y representar estratégicamente los asuntos de los derechos humanos para sus miembros" (KECK y SIKKINK, 2001).

Localizamos en torno del tema de la Defensa y promoción de los derechos humanos en México el surgimiento y desarrollo de los actores a raíz de la política de desaparición selectiva de militantes de la guerrilla urbana y rural fue el Frente Nacional Contra la Represión (FNCR). Para el Dr. Enrique González Ruiz, miembro del Frente Nacional de Abogados Democráticos (FNAD), uno de los fundadores de este frente, "la lucha por los derechos humanos se inicia en el país con la idea de enfrentar la respuesta del Estado al surgimiento de organizaciones insurgentes" (GONZÁLEZ, 2000). A esta etapa de la historia reciente del país, se le ha denominado "guerra sucia" del Estado frente a los grupos radicalizados de la sociedad, como respuesta por parte de esta última se dio la creación de organismos independientes que se dedicaron a la defensa de los presos, los perseguidos y los exiliados políticos. 


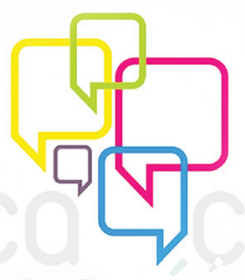

açãô I) Midiática

N. 6 | Ano 2013

Universidade Federal do Paraná | Programa de Pós-Graduação em Comunicação

amplio reconocimiento internacional fue Amnistía Internacional, formada en los sesenta. AI tomó algunas decisiones tácticas clave que sirvieron para enmarcar y representar estratégicamente los asuntos de los derechos humanos para sus miembros” (KECK y SIKKINK, 2001).

Las OSC mexicanas al igual que como lo habíamos registrado en la década anterior con el grupo de Rosario Ibarra, seguían siendo fuertemente influenciadas por sus pares de otras partes del mundo. Keck y Sikkink consideran que Amnistía Internacional (AI) fue el terreno de prueba de toda una nueva generación de activistas en todo el mundo, en particular Mariclaire Acosta, quien fungió durante los dos primeros años de la administración del entonces Presidente Fox, como subsecretaria para Derechos Humanos de la SRE, señala

\begin{abstract}
Obviamente toda mi capacitación y toda mi perspectiva de los derechos humanos provienen de Amnistía. Parece que todos los de la primera y segunda generación de Amnistía Internacional somos como una pequeña mafia. Todos nos conocíamos y nos queríamos mucho, y ahora no hemos repartido por el mundo haciendo otras labores por los derechos humanos. Fue como si explotara una estrella. Se transformó en una galaxia desde muchos puntos de vista. Unos están haciendo trabajo académico en los derechos humanos, o están iniciando su propia ONG, o trabajan por los derechos indígenas, los derechos de la tierra, los derechos de los niños, los derechos de las mujeres. Me parece que Amnistía Internacional era maravillosa, porque en realidad permitió que muchas personas de todo el mundo cobraran conciencia de los derechos humanos (KECK y SIKKINK, 2001).
\end{abstract}

Para las investigadoras Margaret Keck y Kathryn Sikkink, la situación política y de derechos humanos (DH) en México era distinta a la de otros países como Argentina, es decir, aquí no había una dictadura encabezada por militares. Tampoco se cometían los atropellos en masa que allá se registraban, en lo concerniente al número, sin embargo estaban las denuncias planteadas por el Comité Eureka y el FNCR, donde se documentaban cerca de 500 desapariciones forzadas en el contexto de una guerra de baja intensidad en contra del movimiento guerrillero, violaciones de DH que fueron llevadas a cabo por los cuerpos de seguridad del Estado mexicano, a pesar de esto la atención internacional no tomaba en cuenta la situación de los DH en México.

Ellas plantean que seguramente en ello influyó: a) que existía un gobierno civil y "electo"; b) la postura del gobierno mexicano frente al golpe de Estado perpetrado por Augusto Pinochet, en Chile y el asilo que se le brindó a los miles de exiliados de ese país y de otros de la región; c) a su fuerte crítica a las violaciones de DH en El Salvador; d) el desconocimiento y peor aún, el desinterés de la población en el tema y por último; e) la escasa presencia, fuerza y número de organizaciones mexicanas en pro de los DH (KECK y SIKKINK, 2001).

Para el FNCR, la detención desaparición era una práctica usada en todo opositor, disidente político o participante en luchas y reivindicaciones, económicas, políticas, 


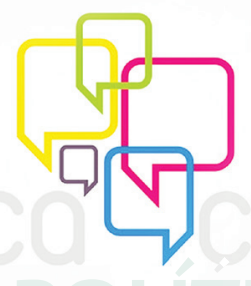

açãô Imidiática

N. 6 | Ano 2013

Universidade Federal do Paraná | Programa de Pós-Graduação em Comunicação

democráticas o sociales, cuyo paradero aún se desconoce, recluido en sitios clandestinos, sujeto a incomunicación, tortura y malos tratos (FNCR, 1979). Vale destacar que de los más de 557 desaparecidos documentados por el Comité Eureka y el FNCR aproximadamente a 400 se les vinculaba oficialmente como participantes en algún movimiento armado.

\section{Los años noventa: la pluralidad de actores}

Desde los años setenta registramos la creación de redes a través de las denominadas coordinadoras, las hubo en torno a lo urbano-popular (CONAMUP), en el campo, donde se enlazaron los temas indígenas (CNPA), hasta que éstos cobran su propio carácter en los 90 con el zapatismo. Otra vertiente fue la sindical (COSINA, CNTE). Así también, en esa década en torno al género (MFM), es decir, registramos la confluencia entre redes en torno a diversas temáticas e intereses comunes.

Una década atrás, en los años ochenta verán surgir la red de organizaciones y actores de la SC en torno a la defensa y promoción de los derechos humanos (FNCR), de los refugiados centroamericanos, feministas y en torno a lo electoral en general (Alianza Cívica).

La década de los noventa, verá el mayor desarrollo de organizaciones, tal y como lo hemos señalado, así también de las redes de actores de la SC. Un sin fin de temáticas, en lo que a nosotros nos ocupa, es decir, las redes que realizan política de la influencia, que se relacionan con el poder político, buscan transformaciones del régimen, autoritario a democrático, vimos surgir entre otras, en 1994 la Alianza Cívica y en 1999 el Poder Ciudadano.

Estas redes, sumadas a las que surgieron en torno al tema de la defensa y promoción de derechos humanos, así como las feministas, apuntamos a que marcaron la década de los noventa. Posteriores al año dos mil han traspasado la década, incluso el milenio todas, incluidas las coyunturales, como Alianza Cívica, en torno de la defensa del voto.

\section{Bibliografia}

Cadena Roa, Jorge (2004), “¿Qué hay de nuevo con las redes mexicanas de organizaciones civiles?" en Las organizaciones civiles mexicanas hoy, México, Centro de Investigaciones Interdisciplinarias en Ciencias Sociales y Humanidades - Universidad Nacional Autónoma de México, pp. 155-187.

Campos B. Rosario (2003), Políticas con Perspectiva de Género en México: análisis de las Redes de políticas de violencia Familiar en el D.F., Guanajuato y Puebla, (Tesis doctoral), México, FCPyS-UNAM.

González Ruiz Enrique (2002), miembro del Frente Nacional de Abogados Democráticos (FNAD), entrevista realizada en la Ciudad de México. 
